\title{
The Expected Performance of the ATLAS Inner Detector
}

\author{
Jason LEE*, on behalf of the ATLAS collaboration. \\ University of Sydney \\ E-mail: jlee@physics.usyd.edu.au
}

\begin{abstract}
The ATLAS experiment [1] is a general purpose detector designed to exploit the discovery potential of the Large Hadron Collider (LHC) at CERN, which will provide proton-proton collisions at a center of mass energy of $14 \mathrm{TeV}$. The Inner Detector will see of the order of 1000 charged particle tracks for every bunch crossing at the design luminosity of the LHC. A summary of the expected performance for reconstructing single particles is presented, along with some measures of the vertex resolution of the vertexing capabilities. The studies focus on the performance expected for the initial running at the start-up of the LHC.
\end{abstract}

European Physical Society Europhysics Conference on High Energy Physics

July 16-22, 2009

Krakow, Poland

\footnotetext{
* Speaker.
} 


\section{Introduction}

The expected performance of the ATLAS Inner Detector (ID) has been studied with a GEANT simulation of the complete detector, complemented by experience gained from test beam and cosmic ray data. Performance definitions have been standardised by the tracking performance group [2] and some of the key points are discussed below.

\section{Tracking Performance}

The ATLAS tracking software [3] is modular in design, enabling multiple tracking strategies with standardised interfaces to all reconstruction tools. Track reconstruction in the ID is logically sub-divided into three stages: raw data from the 3 sub-detectors are transformed into space-points; then the tracks are reconstructed in 2 directions inside-out and outside-in (TRT-segment based pattern recognition, for tracks originating later inside the tracker); and the final processing stage finds primary vertices, then secondary vertices and photon conversions.

The performance of track finding and fitting is shown in Fig. 1. Pions are affected by hadronic interactions in the ID material, while electrons are subject to even larger reconstruction inefficiencies which arise from the effects of bremsstrahlung. As a result, the performance of the ID (resolutions of track parameters and efficiency of track finding) vary as a function of $|\eta|$, which reflects the amount of material in the inner detector.
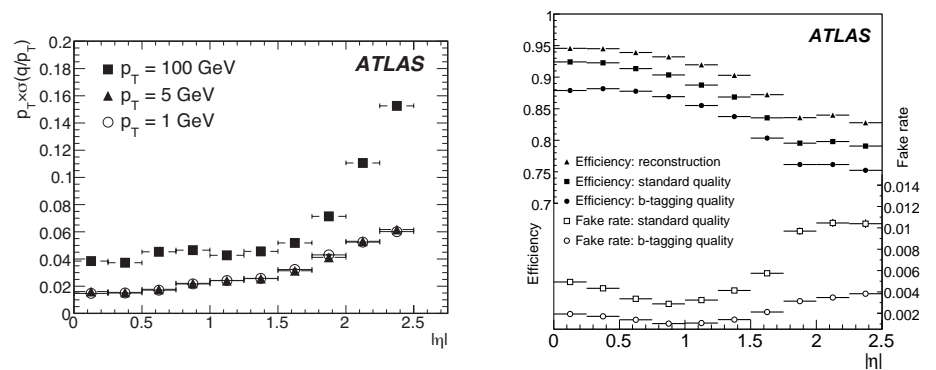

Figure 1: (left) Shows the relative transverse momentum resolution as a function of $|\eta|$ for muons; (right) shows the track reconstruction efficiencies and rate of fake tracks as a function of $|\eta|$ for pions.

\section{Vertex Reconstruction Performance}

Vertexing tools are important for many physics analyses. Figure 2 shows the performance of primary and secondary vertex reconstruction. The primary vertex reconstruction has been tested with $H \rightarrow \gamma \gamma$ and $t \bar{t}$ Monte Carlo events. The resolutions for secondary vertex reconstruction are best for decays just in front of the detector layers. The barrel pixel layers are at radii 51, 89 and $123 \mathrm{~mm}$; the first two SCT layers are at 299 and $371 \mathrm{~mm}$.

\section{Electrons and Photons}

The ATLAS ID faces greater problems for electrons and photons [4] than previous experiments due to the large amount of material within the tracking detectors resulting in greater numbers of 

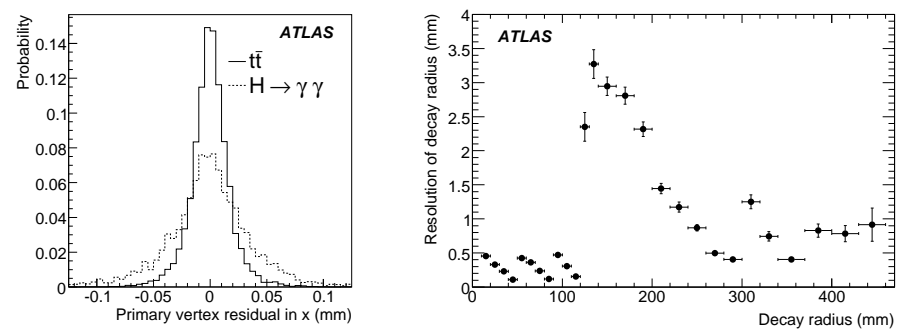

Figure 2: (left) Shows the primary vertex residual along $x$ (transverse plane) for events containing topquark pairs and $H \rightarrow \gamma \gamma$ decays with $m_{H}=120 \mathrm{GeV}$. (right) Shows the resolution for the reconstructed radial position of the secondary vertex for $K_{S}^{0} \rightarrow \pi^{+} \pi^{-}$decays in events containing B-hadron decays as a function of the $K_{S}^{0}$ decay radius. Features due to the various detector layers are evident: see the text.

radiation lengths of material traversed before reaching the calorimeters: 0.4 radiation lengths in the barrel ( 1.1 in the endcaps). This increases the likelihood of electrons losing significant amounts of energy to bremsstrahlung and photons converting within the Inner Detector.

ATLAS employs two bremsstrahlung recovery track fitting algorithms, Gaussian Sum Filter [5] and Dynamic Noise Adjustment [6], to improve the track fitting of electrons. Photon reconstruction takes advantage of both inside-out and outside-in track finding methods and vertex reconstruction, to identify photons that convert later in the ID. The performance of electron and photon reconstruction is shown in Fig. 3.
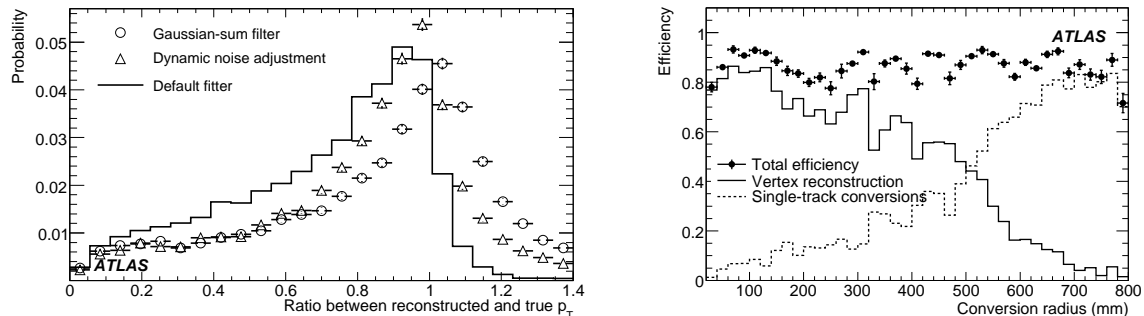

Figure 3: (left) Shows the probability distributions for the ratio of the true to reconstructed momentum for electrons with $p_{T}=25 \mathrm{GeV}$ and $|\eta|>1.5$. (right) Shows the efficiency to identify conversions of photons with $p_{T}=20 \mathrm{GeV}$ and $|\eta|<2.1$, as a function of the conversion radius. The overall efficiency is a combination of the efficiency to reconstruct the conversion vertex and of that to identify single-track conversions.

\section{Summary}

This document is a very brief outline of the performance expected from first data from the LHC. The ID hardware and software has been tested with simulated, cosmic and test beam data; and the barrel section has been aligned with the cosmic data. It is ready to be fully commissioned with first data.

\section{References}

[1] The ATLAS Collaboration, The ATLAS Experiment at the Large Hadron Collider, 2008, JINST 3 S08003 
[2] The ATLAS Collaboration, The Expected Performance of the Inner Detector, Internal report, 2009, ATL-PHYS-PUB-2009-002

[3] T. Cornelissen et al., Concepts, Design and Implementation of the ATLAS New Tracking (NEWT), Internal report, 2007, ATL-SOFT-PUB-2007-007

[4] Elsing, M et al., ATLAS Detector Paper Back-Up Note: Electrons and Photons, Internal Report ATL-INDET-PUB-2008-013, CERN, Geneva, Jul 2008

[5] R. Fruhwirth, A. Strandlie, Track finding and fitting with the Gaussian-sum Filter, 1998

[6] V. Kartvelishvili, Electron bremsstrahlung recovery in ATLAS, Proceedings of the 10th Topical Seminar on Innovative Particle and Radiation Detectors (IPRD06), 2007 